\title{
Sexual dysfunction in dialytic patients. A prospective cross-sectional observational study in two hemodialysis centers
}

\author{
Carlo Pavone $^{1}$, Antonio Simone Di Fede ${ }^{1}$, Piero Mannone ${ }^{1}$, Gabriele Tulone ${ }^{1}$, Arjan Bishqemi ${ }^{1}$, \\ Alberto Abrate ${ }^{2}$, Vincenzo La Milia ${ }^{3}$, Vincenzo Serretta ${ }^{1}$, Alchiede Simonato ${ }^{1}$ \\ ${ }^{1}$ Department of Surgical, Oncological and Oral Sciences, Section of Urology, University of Palermo, Palermo, Italy; \\ ${ }^{2}$ Department of Surgery, Urology Unit, ASST Valtellina e Alto Lario, Sondrio, Italy; \\ ${ }^{3}$ Nephrology and Dialysis Department, Lecco, Italy.
}

\begin{abstract}
Summary Objectives: Incidence and prevalence of patients in dialytic therapy increased considerably in recent years. The onset of new issues, once overshadowed, linked to a lower quality of life like sexual dysfunction became increasingly common. The first study in this area, dating back to the 1970s, shows the high prevalence of sexual dysfunction among patients in dialytic therapy of both sexes. Later studies proved an association of sexual dysfunction with psyche disorders, anxiety, depression and lack of self-confidence. The aim of this study is to describe the incidence of male and female sexual main dysfunctions, the latter not least in literature, in patients in hemodialytic therapy. With this aim two dialytic centers have been compared, one located in northern Italy and one in southern Italy, and the different prevalence has been compared to the general population.
\end{abstract}

Methods: We conducted a prospective cross-sectional observational study in patients undergoing dialytic therapy in two hemodialysis centers, one located in Palermo and one in Lecco. Male sexual dysfunction was investigated by the International Index of Erectile Function-15 (IIEF15) questionnaire and the Premature Ejaculation Diagnotic Tool (PEDT) questionnaire, and the female dysfunction by Female Sexual Function Index (FSFI) questionnaire. Criteria for inclusion in our study were: age $<75$ years and dialytic age $>3$ months; exclusion criteria were: advanced cancer diseases, life expectancy $<6$ months, previous urological manipulation, anti-androgenic therapy, sexual dysfunction unrelated to kidney disease, psychiatric disorders. Data were compared with mean-standard deviation (SD) and with the variance analysis (ANOVA). A value of $p<0.05$ is considered significant. Discrete data were analyzed with contingency analysis. A chi $2<0.05$ was considered significant. Results: Data of 78 patients have been collected. Mean age and dialytic time were $54 \pm 12$ years and $42 \pm 35$ month; 33 patients were from Palermo and 24 from Lecco; 21 patients were exclud$e d$. Age and dialytic age of the two subgroups did not demonstrate statistically significant differences. Between the two centers there was a statistically significant difference $(p<0.005)$ in the distribution of basic nephropathy: an higher incidence of diabetic and obstructive nephropathy has been observed in the southern center compared to northern center, while glomerulonephritis and polycystic kidney disease had an higher incidence in the northern center compared to southern one. The main sexual dysfunctions in both sexes, erectile dysfunction (ED) and premature ejaculation (PE) in men and orgasm disorder and pelvic pain in women, have been investigated. ED was present in $70 \%$ of hemodialyzed patients, which is an higher incidence compared to the general population. The severity of $\mathrm{ED}$ between patients of the two groups was significantly different (chi2 < 0.001) with higher incidence of moderate/severe forms in northern Italy. The score, in addition to discrete data (severe, moderate, mild, absence), of ED was significantly different $(p<0.001)$ between patients of the two centers $(22 \pm 7$ Palermo vs. $9 \pm 8$, Lecco). The PE was absent in 20 patients (54\%), present in 12 patients (32\%) and probable in 5 patients (14\%) (scores of $7.6 \pm 4.0$ and $8.9 \pm 6.8$, respectively in Palermo and Lecco patients). For women, orgasmic dysfunction was severe in 10 patients (50\%), mild in 4 patients (20\%), very mild in 5 patients (25\%), while it was normal in 1 patient $(5 \%)$, with a statistically significant difference $(p<0.05)$ between Palermo and Lecco patients $(3.0 \pm 1.4$ vs $1.2 \pm 2.0)$. Sexual pain in women was severe in 11 patients (55\%), moderate in 4 patients (20\%) and mild in 5 patients $(25 \%)$. Sexual pain was present in all patients $(p<0.05)$.

Conclusions: Regardless of sex, sexual dysfunction is one of the most common side effect in patients with end stage renal disease in dialytic therapy. Our study confirms literature data. The growing number of the dialytic population with sexual disorders needs specialist support to improve quality of life of these patients.

KEY WORDS: Dialysis; End-Stage Renal Disease; Sexual male dysfunction; Erectile disorder; Premature ejaculation; Female sexual dysfunctions; International Index of Erectile Function; Premature Ejaculation Diagnostic Tool.

Submitted 4 November 2020; Accepted 28 December 2020

\section{INTRODUCTION}

End-Stage Renal Disesase (ESRD) is a disabling disease that forces patients to a radical change of their lifestyle and habits, causing physical and important psychological disorders. Sexual male dysfunction is an extremely common condition that manifests itself with erectile disorder (ED) and premature ejaculation (EP) (1), often present at the same time. In everyday clinical practice, both pathologies require a multidisciplinary approach in the diagnostic phase and in the therapeutic management $(2,3)$ due to their multifactorial genesis, such as psychological factors and endothelial dysfunction. Female sexual dysfunctions 
are understudied, despite the incidence being alarmingly high: in a 30 years ago study, $76 \%$ of women were living with some symptoms of sexual dysfunction (4), while more recent literature suggest that prevalence is around $43 \%$ (5). Most common symptoms in women are dyspareunia, vaginismus, and noncoital sexual pain disorder (6). Since population undergoing hemodialysis (HD) treatment for ESRD significantly increased in the last few years, attention is focused on its sexual disease (7).

The greater longevity of these patients and the association with dialytic therapy has led to new problems that in the past were not highlighted in an incisive way and that today have been noticed to have a significant influence on the quality of life among ESRD patients.

First studies on sexual dysfunction in dialysis patients, date back to the 1970s, highlighted the high prevalence of sexual dysfunction among patients of both sexes (8). Following studies confirmed that sexual dysfunction is very common in men and women with ESRD, associated with anxiety and depression, affecting the quality of life with impact on self-confidence, self-esteem and selfimage $(9,10)$. The aim of this study is to describe the incidence of male and female main sexual dysfunctions, in patients undergoing hemodialytic therapy, considering modern dialytic techniques. Two dialytic centers have been compared, one in Northern Italy and another one in Southern Italy. Different prevalence has been compared to the general population.

\section{Methods}

A prospective cross-sectional observational study amongst two hemodialysis centers has been conducted. The presence of sexual dysfunctions has been assessed by questionnaires administered to patients on chronic haemodialysis treatment in both examined haemodialysis centers, one in Southern Italy (Palermo) and one in Northern Italy (Lecco). Questionnaires were administered and returned anonymously. Andrological or gynecological evaluation was offered when requested by patients. Inclusion criteria are age $<75$ years and dialytic age $>3$ months. Exclusion criteria are advanced neoplastic diseases, life expectancy $<6$ months, radical prostatectomy, anti-androgenic therapy, sexual dysfunctions unrelated to kidney disease, any previous surgical manipulation of the urogenital system, use of drugs to improve sexual performance, psychiatric disorders, and people who refused to participate to the study.

Dialytic patients were recruited for the study regardless of the cause that led to end-stage renal failure. Data were collected from January 2018 to December 2018. Questionnaires administered to male patients are International Index of Erectile Function 15 (IIEF) 15 (11) and Premature Ejaculation Diagnostic Tool (PEDT) (12). The Female Sexual Function Index test (FSFI) (13) was administered to Female patients.

Data are compared with mean standard deviation (SD) and with the variance analysis (ANOVA). A value of $\mathrm{p}<$ 0.05 is considered significant. Discrete data are analysed with contingency analysis. A Chi ${ }^{2}<0.05$ value is considered significant. In our study, the sample size was not calculated and this represents a limitation for results.

\section{RESULTS}

78 patients were recruited into the study: 5 of them were excluded for psychiatric issues, 6 refused to answer and 10 did not complete the questionnaire. Therefore, our study was carried out on 57 patients. Patients underwent chronic hemodialysis treatment, aged $54 \pm 12$ years, 37 males and 20 females, with dialysis age $42 \pm 35$ months, of which 33 patients were hemodialyzed in a dialysis center in Southern Italy (Palermo) and 24 patients in a dialysis center in Northern Italy (Lecco).

In the dialysis center of Palermo, 23 male patients were recruited. Patient ages range from a minimum of 25 to a maximum of 72 years, with an average of $54 \pm 14$ years. We also analyzed the dyalisis ages of patients from a minimum of 4 to a maximum of 84 months, with an average of $37 \pm 24$ months. In the dialysis center of Lecco, 14 male patients were recruited. Patient ages range from a minimum of 36 to a maximum of 70 years, with an average of $55 \pm 10$ years. We also collected informations about the dyalisis ages of patients ranging from a minimum of 5 to a maximum of 106 months, with an average of $45 \pm 33$ months. Furthermore, we paid attention to the anamnesis and chronic pathologies that led patients to need dialysis treatment in the respective center (Table 1). The average age of male patients on hemodialysis treatment in Palermo is not significant different from those in Lecco $(54 \pm 14$ years vs $55 \pm 10$ years, respectively) and there is not significant difference in dialysis age, either $(37 \pm 24$ months vs $45 \pm 33$ months, respectively). On the other hand, there is a difference in the most common underlying disease between the two centers: diabetic nephropathy (39\%) in Palermo and polycystic kidney (29\%) in Lecco.

In the dialysis center of Palermo, 10 female patients were recruited. The ages of the patients range from a minimum of 39 to a maximum of 71 years, with an average of $54 \pm 11$ years. $60 \%$ (n. 6) of these patients were in menopause. We also collected informations about the dyalisis ages of the patients ranging from a minimum of 10 to a maximum of 72 months, with an average of $35 \pm$ 20 months. In the center of Lecco, 10 female patients were recruited. The ages of the patients range from a minimum of 34 to a maximum of 63 years, with an average of $53 \pm 10$ years. $80 \%$ (n. 8) of these patients were in menopause. We also collected the dyalisis ages of the patients ranging from a minimum of 9 to a maximum of 209 months, with an average of $57 \pm 62$ months.

\section{Table 1.}

Cause of ESRD.

\begin{tabular}{|lcccc|}
\hline Cause of ESRD & $\begin{array}{c}\text { Male } \\
\text { Palermo }\end{array}$ & $\begin{array}{c}\text { Male } \\
\text { Lecco }\end{array}$ & $\begin{array}{c}\text { Female } \\
\text { Palermo }\end{array}$ & $\begin{array}{c}\text { Female } \\
\text { Lecco }\end{array}$ \\
\hline Hypertensive nephropathy & $3(13 \%)$ & 0 & $1(10 \%)$ & 0 \\
\hline Diabetic nephropathy & $9(39 \%)$ & $3(21 \%)$ & $4(40 \%)$ & $2(20 \%)$ \\
\hline Obstructive nephropathy & $3(13 \%)$ & $1(7 \%)$ & $1(10 \%)$ & 0 \\
\hline Glomerulonephritis & $4(17 \%)$ & $2(14 \%)$ & $1(10 \%)$ & $3(30 \%)$ \\
\hline Polycystic kidney & $1(4 \%)$ & $4(29 \%)$ & $1(10 \%)$ & $4(40 \%)$ \\
\hline Recurrent urinary tract infections & $3(13 \%)$ & $1(7 \%)$ & $2(20 \%)$ & 0 \\
\hline Nephroangiosclerosis & 0 & $3(21 \%)$ & 0 & $1(10 \%)$ \\
\hline Tot & 23 & 14 & 10 & 10 \\
\hline
\end{tabular}


We also paid attention to the anamnesis and chronic pathologies that led patients to need dialysis treatment in the respective center (Table 1). The average age of female patients on hemodialysis treatment in Palermo is not significant different from those in Lecco $(54 \pm 11$ years vs $53 \pm 10$ years, respectively) while there is a significant difference in dialysis age, either ( $35 \pm 20$ months vs $57 \pm$ 62 months, respectively). As in the case of male patients, there is a difference in the most common underlying disease between the two centers: diabetic nephropathy (40\%) in Palermo and polycystic kidney (40\%) in Lecco. ESRD influence on sexual function is analyzed using questionnaires. For male patients following parameters are taken into account: erectile dysfunction, orgasmic dysfunction, libido reduction, dissatisfaction of sexual relations and premature ejaculation (Table 2).

Erectile dysfunction is present in $70 \%$ of patients with different severity level distributed with a significant difference $\left(\mathrm{Chi}^{2}<0.001\right)$. Data show prevalence of severe dysfunction among patients on hemodialysis treatment at the center of Lecco, while at the hemodialysis center of Palermo this dysfunction is absent in most patients.

We documented a different score $(p<0.001)$ among patients of the center of Palermo compared to those of

\section{Figure 1.}

Male's main sexual dysfunctions.

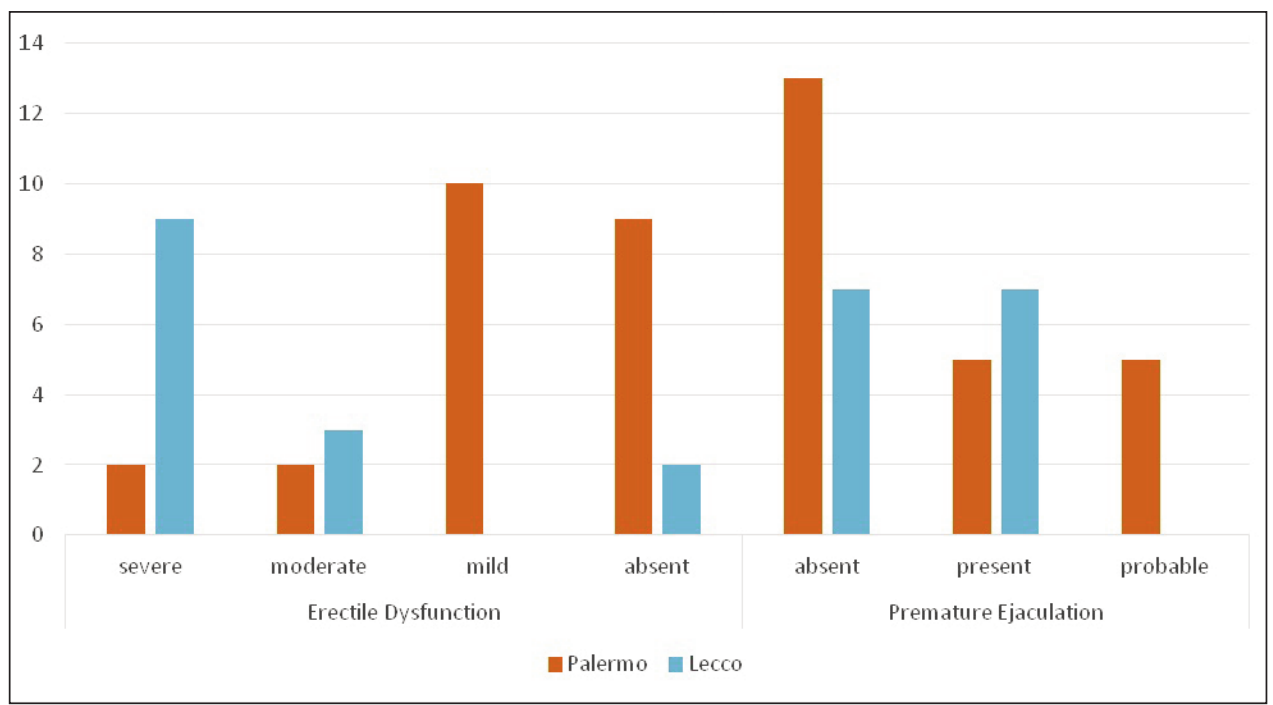

the center of Lecco $(9 \pm 2$ vs $3 \pm 4$, respectively). The reduction in sexual desire is absent in 2 patients (5\%), very slight in 5 patients (14\%), mild in 19 patients (51\%), moderate in 8 patients (22\%) and severe in 3 patients $(8 \%)$. Libido reduction is statistically different $\left(\mathrm{Chi}^{2}<0.05\right)$ between the male patients of the two hemodialysis centers, with a clear prevalence of severe and moderate dysfunction among the patients on hemodialysis treatment at the center of Lecco, whereas it is mild in most patients in treatment at the hemodialysis center in Palermo. We documented a significantly different score ( $\mathrm{p}<0.05)$.

The general sexual well-being is also statistically different $\left(\mathrm{Chi}^{2}<0.05\right)$ with a clear prevalence of severe and moderate impairment among the patients on hemodialysis treatment at the center of Lecco, while this impairment is mild or very mild in most patients in treatment at the hemodialysis center in Palermo. We documented significantly different score $(\mathrm{p}<0.05)$ among patients of the center of Palermo, compared to those of the center of Lecco ( $7 \pm 0$ vs $5 \pm 1$, respectively).

There is a statistically significant difference $\left(\mathrm{Chi}^{2}=0.03\right)$ regarding premature ejaculation: this alteration of sexuality is more frequent in male patients on dialysis at the center of Lecco, compared to male patients of the hemodialysis center in Palermo.

However, when considering the total score of responses on premature ejaculation, instead of considering the discrete variables (present, absent and probable), this difference is no longer significant; in particular, the total score is $7.6 \pm 4.0$ and $8.9 \pm 6.8$ in patients being treated at the hemodialysis center in Palermo and Lecco, respectively (Figure 1) (Table 2).

Table 2.

Males' sexual dysfunctions.

\begin{tabular}{|c|c|c|c|c|c|c|c|c|c|c|c|c|c|}
\hline Patients features & \multicolumn{13}{|c|}{$\begin{array}{l}\text { Patients (n.) } 57 \\
\text { Males: } 37 \text { - Females: } 20 \\
\text { Age (years) } 54 \pm 12 \text { (Palermo } 54 \pm 14 \text {; Lecco } 55 \pm 10) \\
\text { Dialysis age (month) } 42 \pm 35 \text { (Palermo } 37 \pm 24 \text {; Lecco } 45 \pm 33\end{array}$} \\
\hline \multicolumn{14}{|l|}{ Males sexual disfunctions } \\
\hline & \multicolumn{6}{|c|}{ Palermo } & \multicolumn{6}{|c|}{ Lecco } & \multirow[b]{2}{*}{$P$ value } \\
\hline & Severe & Moderate & Mild & Light & Very light & Absent & Severe & Moderate & Mild & Light & Very light & Absent & \\
\hline Orgasmic dysfunction (\%) & $1(4.3 \%)$ & & $1(4.3 \%)$ & & $2(8.7 \%)$ & $19(82.7 \%)$ & $9(64.3 \%)$ & & 0 & & $3(21.5 \%)$ & $2(14.2 \%)$ & $<0.001$ \\
\hline Libido reduction (\%) & 0 & & $3(13 \%)$ & $15(65.3 \%)$ & $3(13 \%)$ & $2(8.7 \%)$ & $3(21.5 \%)$ & & $5(35.8 \%)$ & $4(28.5 \%)$ & $2(14.2 \%)$ & 0 & $<0.05$ \\
\hline \multirow[t]{2}{*}{ Dissatisfaction of sexual relations (\%) } & $2(8.7 \%)$ & & 0 & $12(52.1 \%)$ & $9(39.2 \%)$ & & $10(71.4 \%)$ & & $2(14.2 \%)$ & $1(7.1 \%)$ & $1(7.1 \%)$ & & $<0.001$ \\
\hline & Present & & Probable & & & Absent & Present & & Probable & & & Absent & \\
\hline Premature ejaculation (\%) & $5(21.7 \%)$ & & $5(21.7 \%)$ & & & $13(56.6 \%)$ & $7(50 \%)$ & & 0 & & & $7(50 \%)$ & \\
\hline
\end{tabular}


Here below female patients' data were then analyzed. Through the scores we investigated parameters such as reduction of sexual desire, reduction of sexual arousal, lubrication, orgasmic dysfunction, sexual disatisfaction and sexual pain (Table 3). The reduction in sexual desire is statistically significant $\left(\mathrm{Chi}^{2}<0.05\right)$ in female patients in hemodialysis treatment in the two hemodialysis centers, with it being severe or moderate in patients in hemodialysis treatment in Lecco, while in patients in hemodialysis treatment in Palermo it presented in a milder form. However, the analysis of the score, i.e. the continuous variable, do not show a statistically significant difference between patients of Palermo and those of Lecco $(2.2 \pm 0.9$ vs $2.8 \pm 0.9)$.

The reduction in sexual arousal is not statistically significant in female patients in hemodialysis treatment at the two hemodialysis centers, although the reduction in sexual arousal is more severe in hemodialysis patients in Lecco than in hemodialysis patients in Palermo. The score shows a statistically significant difference $(\mathrm{p}<0.005)$ between patients of Palermo and those of Lecco (3.2 \pm 1.1 vs $1.3 \pm 1.7$ ) with a greater dysfunction of sexual arousal in the latter. The reduction in lubrication is statistically significant $\left(\mathrm{Chi}^{2}<0.01\right)$ in female patients in hemodialysis treatment at the two hemodialysis centers, being severe or moderately severe among patients in hemodialysis treatment in Lecco, while in patients in hemodialysis treatment in Palermo it manifested itself in a milder form.

\section{Figure 2.}

Female's main sexual dysfunctions.

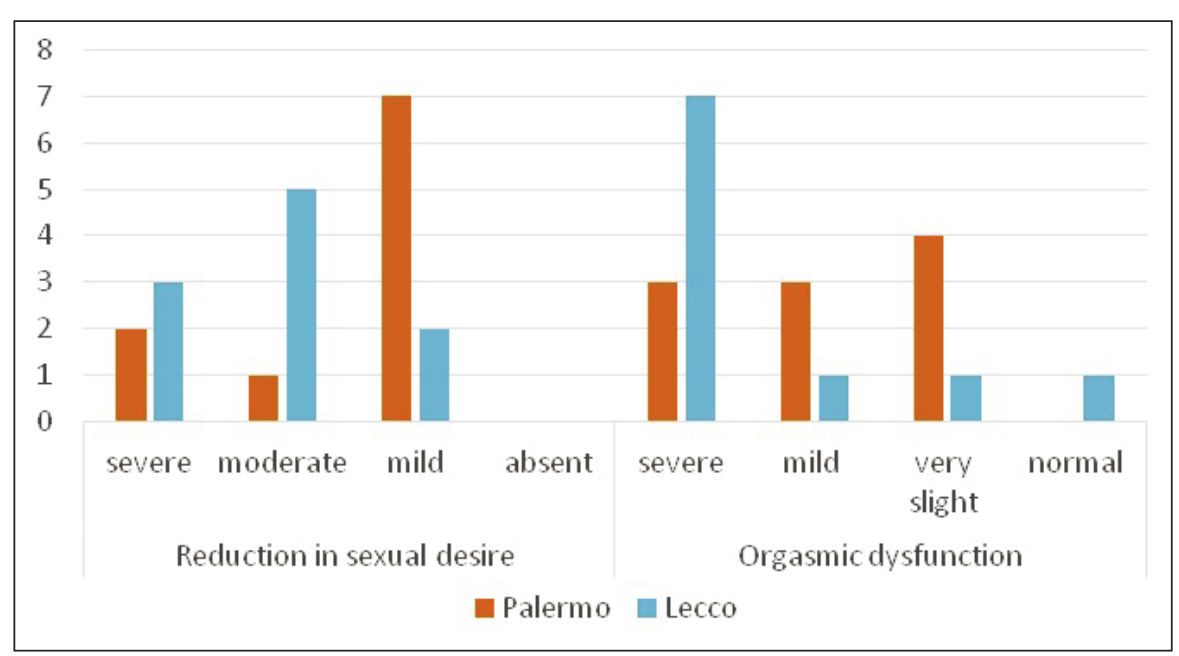

The analysis of the score, i.e. the continuous variable, do not show a statistically significant difference between the patients of Palermo and those of Lecco (3.2 \pm 1.2 vs $1.5 \pm$ 2.5 , respectively).

Orgasmic dysfunction is not statistically significant in female patients in hemodialysis treatment at the two hemodialysis centers, although there is a greater tendency for severe dysfunction in patients in hemodialysis treatment at the center of Lecco than in patients in hemodialysis treatment in Palermo. The score shows a statistically significant difference $(\mathrm{p}<0.05)$ between the patients of Palermo and those of Lecco $(3.0 \pm 1.4$ vs $1.2 \pm 2.0$, respectively). Sexual dissatisfaction is statistically significant $\left(\mathrm{Chi}^{2}<0.001\right)$ in female patients in hemodialysis treatment at the two hemodialysis centers, with a greater tendency for severe sexual dissatisfaction in patients in hemodialysis treatment at the center of Lecco, compared to patients in treatment hemodialysis in Palermo.

The analysis of the score, i.e. of the continuous variable, also shows a statistically significant difference $(\mathrm{p}<0.001)$ between the patients of Palermo and those of Lecco (3.8 \pm 0.4 vs $0.7 \pm 0.4$, respectively).

Finally, sexual pain presents a statistically significant difference $\left(\mathrm{Chi}^{2}<0.05\right)$ in female patients in hemodialysis treatment at the two hemodialysis centers, with a greater tendency to severe sexual pain in patients in hemodialysis treatment at the center of Lecco, compared to patients in hemodialysis treatment in Palermo.

The analysis of the score, i.e. the continuous variable, also shows a statistically significant difference $(p<0.05)$ between the patients of Palermo and those of Lecco $(2.2 \pm 0.7$ vs $0.8 \pm 1.8$, respectively) (Figure 2, Table 3).

\section{Discussion}

Analyzing available data, the two main male and female typical sexual dysfunctions of the chronic hemodialytic patient were compared with those present in the general population, also making a comparison with data obtained throughout this study.

\section{Table 3.}

Females' sexual dysfunctions.

\begin{tabular}{|c|c|c|c|c|c|c|c|c|c|c|c|}
\hline \multicolumn{12}{|l|}{ Females sexual disfunctions } \\
\hline & \multicolumn{5}{|c|}{ Palermo } & \multicolumn{5}{|c|}{ Lecco } & \multirow[t]{2}{*}{$P$ value } \\
\hline & Severe & Moderate & Mild & Very mild & Absent & Severe & Moderate & Mild & Very mild & Absent & \\
\hline Reduction of sexual desire (\%) & $2(20 \%)$ & $1(10 \%)$ & $7(70 \%)$ & & & $3(30 \%)$ & $5(50 \%)$ & $2(20 \%)$ & & & \\
\hline Reduction in sexual arousal (\%) & $1(10 \%)$ & $2(20 \%)$ & $5(50 \%)$ & $2(20 \%)$ & 0 & $6(60 \%)$ & $1(10 \%)$ & $2(20 \%)$ & $1(10 \%)$ & 0 & $<0.005$ \\
\hline Female lubrication reduction (\%) & $1(10 \%)$ & $2(20 \%)$ & $2(20 \%)$ & $5(50 \%)$ & 0 & $7(70 \%)$ & 0 & 0 & $1(10 \%)$ & $2(20 \%)$ & \\
\hline Orgasmic dysfunction (\%) & $3(30 \%)$ & & $3(30 \%)$ & $4(40 \%)$ & 0 & $7(70 \%)$ & & $1(10 \%)$ & $1(10 \%)$ & $1(10 \%)$ & $<0.05$ \\
\hline Sexual dissatisfaction (\%) & $1(10 \%)$ & 0 & $3(30 \%)$ & $6(60 \%)$ & & $7(70 \%)$ & $2(20 \%)$ & $1(10 \%)$ & 0 & & $<0.001$ \\
\hline Sexual pain (\%) & $3(30 \%)$ & $4(40 \%)$ & $3(30 \%)$ & & 0 & $8(80 \%)$ & 0 & $2(20 \%)$ & & 0 & $<0.05$ \\
\hline
\end{tabular}


By evaluating erectile dysfunction (ED) we can show how this dysfunction has increased in the chronic hemodialytic population, compared to the general one, as we expected. According to general data, erectile dysfunction in Italy is present in about 3-5 million men, with a prevalence percentage ranging from $10 \%$ to $17 \%$. According to an Italian study by Parazzini et al. (14), the prevalence of ED assessed by questionnaire in the general population was $12.8 \%$, with $70 \%$ partial $\mathrm{ED}$, while $30 \%$ is complete.

These epidemiological data increase in the chronic hemodialytic population, as reported in the literature.

In fact, according to a study by Savadi et al. (15), the prevalence of ED in patients with chronic renal failure was reported in the range of $22-88 \%$ and more specifically it is $87.5 \%$ in patients on hemodialysis. Analyzing our data, ED prevalence of $70 \%$ is observed, considering also the mild form, and it is more frequent in the population of Northern Italy than in that of Southern Italy. Evaluating premature ejaculation, it has been observed that in the Italian general population it is present in about 4-5 million men with a prevalence ranging from about 15 to $20 \%$; while according to the literature, and more precisely according to Aslan, the prevalence of premature ejaculation is determined in $31.6 \%$ of patients (16). Analyzing our data, it was observed that the prevalence of premature ejaculation in our population is $46 \%$.

Assessing the alterations of the female sexual sphere, although data from female hemodialytic patients are very limited, it has been observed that according to one of the largest study carried out worldwide, the prevalence of these disorders is around $84 \%$, confronted with a general population prevalence of about $45 \%$ (more precisely, in the general population there is, for example, a dyspareunia ranging from $12 \%$ in women of childbearing age up to $31 \%$ in those in menopause; or vaginismus that is about $0.5-15 \%$ ) (9). Data reported by our study, however, show that sexual dysfunctions in women receiving hemodialysis are around in 100\% of patients, in almost all assessed items.

Furthermore, comparing data of these sexual dysfunctions between the population of Northern Italy and that of Southern Italy, in the two hemodialysis centers, it is evident that they are different, with a greater prevalence in the Northern population compared to the Southern one. This could be due to different reasons. These differences allow us only to be able to formulate diagnostic hypotheses underlying the different results obtained by our study. A definitely important aspect to explain these differences is basic nephropathy: our data show a significant difference between basic nephropathy in the population assessed in the North and in the South; it cannot be excluded that the underlying pathology may contribute to these differences in sexual dysfunction between the two Italian populations. Even the dialysis age, slightly higher in Lecco patients than in Palermo patients, could explain this difference between the two populations. The cultural differences between the two regions of the country could also have influenced the test results by changing the approach to the questions and altering the answers.

In addition, by assessing the prevalence of male and female sexual dysfunctions, it is evident that these are more frequent in the male than in the female sex. This dif- ference could be due to different pathogenetic factors in the male sex compared to the female one.

Finally, psychological factors in these differences cannot be excluded.

\section{Conclusions}

Our study confirms literature data, according to which chronic renal failure is a disabling disease, also from a sexual functionality point of view, of both male and female patients, and confirms the need for specialist andrological or gynecological advice, in addition to dialysis.

The growing number of dialytic populations with sexual disorders needs specialist support to improve their quality of life.

\section{Conflict of interest statement}

Authors declare that they have no competing interests. The procedures followed were in accordance with the ethical standards of the responsible committee on human experimentation (institutional and national) and with the Helsinki Declaration of 1975, as revised in 2000. Informed consent was obtained from all patients for being included in the study.

\section{REFERENCES}

1. Verze P, Arcaniolo D, Palmieri A, et al. Premature ejaculation among Italian men: prevalence and clinical correlates from an observational, non-interventional, cross-sectional, epidemiological study (IPER). Sex Med. 2018; 6:193-202.

2. Iacona $R$, Bonomo V, Di Piazza M, et al. Five-year prospective study on cardiovascular events, in patients with erectile dysfunction and hypotestosterone. Arch Ital Urol Androl. 2017; 89:313-315.

3. Pavone C, Abbadessa D, Gambino G, et al. Premature ejaculation: Pharmacotherapy vs group psychotherapy alone or in combination. Arch Ital Urol Androl. 2017; 89:114-119.

4. Frank E, Anderson C, Rubinstein D. Frequency of sexual dysfunction in "normal" couples. N Engl J Med. 1978; 299:111.

5. Laumann EO, Paik A, Rosen RC. Sexual dysfunction in the United States: prevalence and predictors. JAMA 1999; 281:537.

6. Allahdadi KJ, Tostes RC, Webb RC. Female sexual dysfunction: therapeutic options and experimental challenges. Cardiovasc Hematol Agents Med Chem. 2009; 7:260-269.

7. McCullough KP, Morgenstern H, Saran R, et al. Projecting ESRD incidence and prevalence in the United States through 2030. JASN. 2019; 30:127-135.

8. Milne JF, Golden JS, Fibus L. Sexual dysfunction in renal failure: a survey of chronic hemodialysis patients. Int J Psychiatry Med. 1977; 8:335-345.

9. Strippoli GF. Collaborative Depression and Sexual Dysfunction (CDS) in Hemodialysis Working Group, Vecchio M, et al. Sexual dysfunction in women with ESRD requiring hemodialysis. Clin J Am Soc Nephrol. 2012; 7:974-981.

10. Edey MM. Male sexual dysfunction and chronic kidney disease. Front Med (Lausanne). 2017; 4:32.

11. Rosen RC, Riley A, Wagner G, et al. The international index of 
erectile function (IIEF): a multidimensional scale for assessment of erectile dysfunction. Urology. 1997; 49:822-830.

12. Symonds T, Perelman MA, Althof S, et al. Development and validation of a premature ejaculation diagnostic tool. Eur Urol. 2007; 52:565-73.

13. Meston CM. Validation of the Female Sexual Function Index (FSFI) in women with female orgasmic disorder and in women with hypoactive sexual desire disorder. J Sex Marital Ther. 2003; 29:39-46.
14. Parazzini F, Menchini Fabris F, Bortolotti A, et al. Frequency and determinants of erectile dysfunction in Italy. Eur Urol. 2000; 37:43-9.

15. Savadi $H$, Khaki M, Javnbakht M, Pourrafiee $H$. The Impact of Hemodialysis on Sexual Function in Male Patients using the International Index of Erectile Function Questionnaire (IIEF). Electron Physician. 2016; 8:2371-2377.

16. Aslan G, Arslan D, Cavdar C, et al. Analysis of premature ejaculation in hemodialysis patients using the International Index of Erectile Function. Urol Int. 2003; 70:59-61.

\section{Correspondence}

Carlo Pavone, MD, Professor (Corresponding Author)

carlo.pavone@unipa.it

Antonio Simone Di Fede, MD

Piero Mannone, MD

Gabriele Tulone, MD

Arjan Bishqemi, MD

Vincenzo Serretta, MD

Alchiede Simonato, MD

Department of Surgical, Oncological and Oral Sciences, Section of Urology, University of Palermo, Palermo (Italy)

Alberto Abrate, MD

Department of Surgery, Urology Unit, ASST Valtellina e Alto Lario, Sondrio, (Italy)

Vincenzo La Milia, MD

Nephrology and Dialysis Department, Lecco (Italy) 